Anne-Cécile Lamy-Joswiak

Faculté des lettres, Université de Ljubljana

Slovénie

anne.cecile.lamy-joswiak@ff.uni-lj.si
UDK 811.133.1'243:37.064.2(497.4)

DOI: $10.4312 /$ vestnik.11.229-250

\title{
REPRÉSENTATIONS DE L'ENSEIGNANT NATIF CHEZ LES APPRENANTS SLOVĖNES AU NIVEAU SECONDAIRE
}

INTRODUCTION

La notion de représentation circule largement dans le champ de la didactique des langues et des cultures (DLC) depuis une vingtaine d'années (Matthey 1997, Zarate \& Candelier 1997, Moore \& Castellotti 2001, Derivry-Plard 2008), et les nombreux travaux relatifs à ce sujet la déclinent en une multiplicité d'objets ${ }^{1}$. Les images et les perceptions que les acteurs sociaux se font d'une langue, de ce que sont ses normes, ses caractéristiques, son statut par rapport à d'autres langues, influencent grandement les processus et les stratégies d'apprentissage qu'ils développent et emploient pour en user (Dabène 1997, Moore 2012). Ce qui relevait auparavant du domaine de la psychologie, comme les attitudes et les comportements, est désormais lié à celui des représentations sociales. Selon Jodelet (1989 : 36), la représentation sociale est « une forme de connaissance socialement élaborée et partagée, ayant une visée pratique et concourant à la construction d'une réalité commune à un ensemble social ». Quant à sa construction et son fonctionnement, Moscovici $(1961,1973)$ lui attribue deux processus : l'objectivation et l'ancrage.

La présente contribution porte sur le poids des représentations de l'enseignant natif/non natif chez des apprenants ${ }^{2}$ allophones dans le contexte de l'enseignement du français langue étrangère (FLE) en Slovénie, où l'intégration des professeurs « étrangers » pour l'enseignement des langues étrangères dans les établissements secondaires date de $1993^{3}$ et s'est achevée en 2015 avec la fin du projet Obogateno učenje tujih

1 Représentations des langues (Candelier \& Hermann-Brennecke 1993, Dabène 1997, Matthey 1997, Castellotti \& Moore 2002), des cultures (Beacco 2001), du bilinguisme (Cavalli \& Coletta 2002), du plurilinguisme (Lüdi \& Py 1995 et 2002), de l'apprentissage (Muller \& de Pietro 2001, Moore \& Castellotti 2001), de l'étranger (Zarate 1993), des enseignants (Causa \& Cadet, 2006).

2 Dans le présent article, l'utilisation du genre masculin pour désigner le groupe enquêté a été adoptée afin de faciliter la lecture et n'a aucune intention discriminatoire.

31993 pour les enseignants natifs d'anglais, 1995 pour les enseignants natifs de français. 
jezikov $v^{4}$ (Apprentissage enrichi des langues étrangères). Celui-ci prévoyait notamment l'enseignement des langues étrangères en tandem (Sodelovalno in timsko poučevanje), autrement dit l'étroite coopération - et donc la présence simultanée en classe - de deux enseignants : l'un natif de la langue source (celle des apprenants), l'autre natif de la langue cible (la langue étrangère à acquérir). Dès lors, se pose la question de savoir comment les apprenants, confrontés à ce tandem original, perçoivent leurs enseignants de langue étrangère, selon leur statut sociolinguistique ; d'où la problématique que soulèvent les notions de locuteur natif et non natif, et tout particulièrement ici, celles d'enseignant de langue natif ou non natif (désormais EN et ENN).

Dans le domaine de l'appropriation des langues, le locuteur natif (désormais LN) se définit par sa compétence linguistique que l'apprenant non natif utilise comme modèle. Pourtant, l'enseignement/apprentissage des langues demeure encombré de la figure du natif, incarnation stéréotypée de la norme et référence inaccessible mais toujours présente sur les différentes grilles d'évaluation (en particulier celles du CECR) et génératrice d'insécurité linguistique chez les apprenants et les enseignants de langue. Les différentes critiques formulées à l'encontre du natif (Paikeday, 1985 ; Renaud, 1998 ; Davies, 1991 et 2003 ; Portine, 2011) permettent de comprendre en quoi il existe un décalage entre l'ambiguïté de la notion - le mythe du locuteur natif - et son implication en didactique des langues et des cultures - l'évaluation des compétences linguistiques par rapport à celle du natif. Dans ce domaine, la légitimité linguistique du natif est désormais largement contestée par les théoriciens du plurilinguisme et de l'interculturel (Byram, 1997 et 2002 ; Beacco, 2005 ; Moore, 2006 ; Coste, 2010). Diverses études francophones (Dabène (dir.) 1990, Causa 2002, Causa \& Vlad 2008, Derivry-Plard 2006, Dervin \& Badrinathan (dir.) 2011) témoignent des représentations des acteurs de l'enseignement/apprentissage des langues étrangères - apprenants, (futurs) enseignants, administrateurs - envers les notions d'EN et ENN, et de la plus ou moins grande légitimité dont jouissent les enseignants natifs face aux non natifs, en fonction des contextes (Derivry-Plard 2008).

Cet article rend compte d'une enquête menée auprès d'un échantillon de 129 lycéens et lycéennes slovènes apprenant le FLE avec un tandem d'enseignantes, l'une native de la langue française, l'autre non. Le questionnaire visait, d'une part, à déterminer la nature et la dynamique des représentations que les apprenants se forgent à l'égard du natif - locuteur et enseignant - et, d'autre part, à comprendre comment ils se positionnent par rapport au statut sociolinguistique de leurs enseignantes de français. L'étude comparative entre

4 Ce projet de 5 ans (2010-2012, 2012-2013, 2013-2015) est le prolongement de deux programmes consécutifs lancés par le Zavod RS za šolstvo (Institut de la République de Slovénie pour l'éducation) en 2004 et financés en partie par le Conseil de l'Europe : Evropski oddelki (Classes européennes) pour la période 2004-2008 et Uvajanja inovativnih pristopov $k$ poučevanju tujih jezikov z vključevanjem tujih učiteljev v izvedbeni kurikulumi (Introduire des approches innovantes pour l'enseignement des langues étrangères en impliquant les enseignants étrangers dans la mise en œuvre des programmes) pour la période 2008-2010. D'ailleurs, certains enseignants étrangers/natifs impliqués dans ces projets sont toujours en poste en Slovénie. 
les niveaux d'apprentissage permettra de déceler les attitudes et les comportements des apprenants en fonction de leurs besoins, de leurs compétences et des objectifs d'apprentissage qu'ils se sont fixés. La conclusion traitera des rapports entre identité linguistique et légitimité professionnelle à l'aune des schèmes de perception à l'œuvre dans le champ de l'enseignement des langues étrangères, les implications de ces représentations et les perspectives de recherches à entrevoir.

\section{RECUEIL DES DONNÉES : DÉMARCHE MÉTHODOLOGIQUE ET OBJECTIFS}

\subsection{Méthodologie de recherche et positionnement}

L'objectif de notre recherche étant de saisir la nature des représentations que les apprenants se forgent à l'égard du natif, nous avons choisi comme point de départ de notre réflexion, le thème de la prétendue « valeur ajoutée » de l'EN, tel que les institutions slovènes l'ont envisagée pour le projet «Apprentissage enrichi des langues étrangères » (Obogateno učenje tujih jezikov). Nous avons voulu connaître les perceptions de nos apprenants à propos de cette « valeur ajoutée ».

Dans l'étude sociologique entreprise, nous posons l'hypothèse que les représentations des apprenants slovènes sur l'EN se modifient en fonction de leur niveau, c'està-dire du développement de leur compétence linguistique et de leur connaissance de la langue/culture cibles. Le choix d'une étude comparative entre les niveaux a été motivé par la relation particulière qu'ils entretiennent avec le tandem EN/ENN au cours des quatre années de leur apprentissage du français. Nous supposons qu'au cours de l'apprentissage, quand le niveau de connaissance de la langue/culture est mieux maîtrisé, les apprenants prennent conscience de leurs savoirs et savoir-faire, autrement dit ils acquièrent une conscience linguistique plus forte, ce qui leur permet de se distancier par rapport à ce que leur transmet l'EN en fonction de leurs besoins, et par conséquent les représentations du modèle à atteindre - celui du natif comme locuteur idéal - sont modifiées. Nous prenons également en compte l'affectivité que les apprenants investissent dans la relation avec l'EN pour en évaluer l'influence sur leurs représentations et leurs éventuelles modifications. Les résultats de l'analyse devraient ainsi permettre de dégager les critères à partir desquels apprendre une LE avec un EN constitue une « valeur ajoutée ».

\subsection{Structure et orientations du questionnaire}

Afin de recueillir chez les apprenants leurs conceptions du natif, locuteur et enseignant, et leurs évaluations quant aux rôles respectifs de leurs enseignantes, native et non native, nous avons opté pour le questionnaire de type sociologique (De Singly, 2005) avec des 
questions fermées sur le parcours des apprenants enquêtés et de leurs enseignantes, et des questions ouvertes portant sur les représentations par rapport à ces dernières.

Pour en constituer la trame, nous nous sommes appuyés sur une recherche du hongrois Medgyes (1992) menée auprès d'enseignants natifs et non natifs d'anglais langue étrangère, et qui s'intéresse aux trois questions suivantes :

1. Qu'est-ce qui définit et distingue un natif d'un non-natif?

2. Les manières d'enseigner d'un professeur natif et d'un professeur non natif sont-elles différentes? Si c'est le cas, cette différence est-elle liée à la compétence linguistique ?

3. Un enseignant natif est-il forcément meilleur qu'un enseignant non-natif en raison de sa compétence linguistique?

Le questionnaire que nous avons élaboré en français (voir en annexe), avant sa traduction en slovène pour qu'il soit soumis aux apprenants, se compose de cinq parties :

1. Toi et l'apprentissage du FLE

2. Le locuteur natif

3. Les enseignants de langue étrangère, natif et non natif

4. Tes rapports avec l'enseignant natif

5. Les cours avec l'enseignant natif

\subsection{Présentation de l'échantillon et conditions de l'enquête}

Nous avons constitué un échantillon suffisamment grand pour apporter des résultats statistiques signifiants. Les données ont été recueillies auprès de 129 lycéens et lycéennes - soit 51,19\% des apprenants de français dans l'établissement secondaire choisi - âgés de 15 à 18 ans ; 121 suivent le cours de français deuxième langue (LV2) de la première à la quatrième année, et 8 apprennent le français comme troisième langue étrangère (LV3), à raison de 3 heures minimum par semaine pour chaque groupe. L'ensemble du groupe interrogé a un profil sociolinguistique relativement homogène : ils partagent tous le slovène comme langue maternelle, l'anglais est leur première langue étrangère (LV1) et ils sont tous en contact avec l'EN au moins une fois par semaine. Quatre groupes ont donc été constitués par année, celui de la deuxième année ayant été dédoublé pour distinguer les apprenants de français LV2 et LV3. Les différences entre ces apprenants sélectionnés pour cette étude relèvent non seulement du niveau d'apprentissage, mais aussi des contenus d'enseignement cumulatifs selon les niveaux et les filières (générale ou européenne). En troisième et quatrième année, des matières optionnelles facultatives et obligatoires augmentent le volume horaire et les contenus d'apprentissage, et donc, le contact avec l'EN. Le tableau suivant indique le nombre et le profil des apprenants interrogés. 


\begin{tabular}{|c|c|c|c|c|c|c|c|c|}
\hline Années d'études & 1 ère année & \multicolumn{2}{|c|}{$2^{\text {ème }}$ année } & \multicolumn{2}{|c|}{$3^{\text {ème }}$ année } & \multicolumn{3}{|c|}{$4^{\text {ème }}$ année } \\
\hline Cours de français & LV2 & LV2 & LV3 & LV2 & $\mathrm{LV} 2+\mathrm{KIC}$ & LV2 & $\mathrm{KIC}$ & Matura $^{5}$ \\
\hline Niveaux du CECR & $\mathrm{A} 1$ & $\mathrm{~A} 2$ & $\mathrm{~A} 1$ & $\mathrm{~A} 2 / \mathrm{B} 1$ & B1 & B1 & $\mathrm{B} 1+$ & $\mathrm{B} 1+$ \\
\hline $\begin{array}{l}\text { Nombre d'élèves } \\
\text { interrogés }\end{array}$ & 44 & 30 & 8 & 20 & 8 & \multicolumn{3}{|l|}{19} \\
\hline $\begin{array}{l}\text { Volume horaire } \\
\text { annuel }\end{array}$ & 105 & 105 & 105 & 105 & $105+70$ & 105 & 35 & $70 / 140$ \\
\hline $\begin{array}{l}\text { Contact horaire } \\
\text { annuel passé } \\
\text { avec I'EN }\end{array}$ & 17,5 & 17,5 & 35 & 17,5 & $17,5+70$ & 35 & 35 & 70 \\
\hline
\end{tabular}

Quant au tandem d'enseignantes, il est composé de deux praticiennes, l'une française (EN), l'autre slovène (ENN), ayant en commun une formation universitaire en langues, littératures et civilisations étrangères (anglais et français) et une formation en didactique des langues et du FLE qu'elles ont acquises dans leurs pays respectifs. Au moment de l'enquête, les deux enseignantes coopèrent étroitement depuis huit ans au sein de ce tandem EN/ENN qui consiste à programmer les contenus d'enseignement, prévoir les pratiques de classes adéquates afin de dispenser les cours de FLE en binôme à tous les niveaux d'apprentissage indiqués ci-dessus.

Le questionnaire - anonyme - a été soumis aux apprenants dans leur langue maternelle, en slovène. La passation s'est tenue sur le temps d'un cours de français de 45 minutes, en présence du tandem EN/ENN, et en fin d'année scolaire afin qu'ils s'appliquent à réfléchir sur l'enseignement reçu au fil de l'année écoulée. Il était important de préparer les apprenants afin qu'ils répondent en confiance. C'est pourquoi en introduction leur ont été précisés les objectifs de cette enquête à finalité de recherche en insistant sur l'intérêt de répondre le plus sincèrement possible, et non par convenance, autrement dit pour « faire plaisir » à l'enseignante/enquêtrice. En effet, connaître le public interrogé et demeurer proche des interviewés a permis tout d'abord, de rencontrer son enthousiasme pour la passation de l'enquête, et ensuite, de recueillir la totalité des questionnaires (129) renseignés avec soin et précision, notamment pour les réponses libres.

\section{3}

\section{RÉSULTATS ET ANALYSES}

Les résultats présentés ci-dessous correspondent à une analyse croisée de données quantitatives et qualitatives (Martin, 2007 ; De Singly, 2008) et synthétisent les réponses aux questions 10 à 20 afin de dégager les représentations de l'EN chez les apprenants slovènes. L'analyse de contenu thématique, retenue pour repérer le nombre et la fréquence

5 Examen de fin d'études secondaires qui sanctionne l'admission aux études supérieures. Le français fait partie des matières optionnelles obligatoires que les candidats peuvent choisir de soumettre à cet examen. 
des occurrences, permet de caractériser les jugements positifs envers l'EN. Comme le précise Derivry-Plard (2011:35), « il s'agit bien des représentations des enquêtés, et non d'une quelconque vérité qu'ils exprimeraient si ce n'est la leur. Autrement dit, les résultats présentent ce que les apprenants pensent de leurs enseignants et leurs catégories de perception sont réduites à « des idéaux-types $»^{6} »$. Dans notre enquête qui insiste sur la relation d'apprentissage avec l'EN, les apprenants adhèrent à l'unanimité à la « valeur ajoutée » de l'EN sans toutefois déprécier le rôle tenu par l'ENN et la sécurité pédagogique qu'il représente dans le processus d'appropriation du français.

\subsection{Perceptions du natif}

Lorsqu'il est demandé aux apprenants de définir le locuteur natif (question 10), force est de constater qu'ils ne parviennent pas à trouver de consensus puisque 14 définitions ont été recensées pour tenter de le caractériser :

1. Celui qui enseigne sa langue maternelle dans son pays d'origine ou à l'étranger.

2. Celui qui parle sa LM (depuis sa naissance).

3. Celui dont la LM est la LE qu'on apprend à l'école.

4. Celui qui parle/maîtrise une langue depuis sa naissance.

5. Celui qui parle la langue du pays où il est né et a grandi/vécu.

6. Celui qui parle sa LM dans un pays étranger.

7. Celui qui est né dans un pays, l'a quitté pour vivre et/ou travailler à l'étranger où il parle sa LM.

8. Celui qui parle la langue officielle du pays où il est né/ de son pays d'origine.

9. Celui dont la LM est le français.

10. Celui qui parle la langue de son pays d'origine.

11. Celui qui vient d'un pays étranger dont il parle la langue couramment.

12. Celui qui parle la langue d'usage d'un pays.

13. Celui dont la LM n'est pas le slovène.

14. Celui qui maîtrise parfaitement une LE.

À partir de ces 14 définitions, on peut dégager deux incarnations du natif : le sujet parlant (« celui qui parle ») d'une part, et le sujet enseignant (« celui qui enseigne ») d'autre part. Hormis la confusion établie entre LN et EN par un tiers des enquêtés $(35,1 \%)$, tous niveaux confondus, ces représentations font émerger deux types de relation avec la langue du natif : une relation d'usage de la langue et une relation d'apprentissage de la langue. Si l'on examine les occurrences du syntagme langue dans les définitions que les

6 « Pour Max Weber, l'idéal-type est un grossissement de l'idéalisation de traits fondamentaux qui forment des « tableaux de pensée homogène ", l'ensemble des traits rassemblés, pas nécessairement les plus courants, mais les plus spécifiques et les plus distinctifs caractérisent l'objet. » (Op.cit.) 
apprenants donnent du LN, on reconnaît le caractère composite que Dabène (1994 : 8-27) attribue à la notion de langue maternelle. Ils semblent faire référence à une diversité de réalités langagières qui empêchent de donner à la langue du natif une définition stable et homogène. Par ailleurs, le nombre et la fréquence d'occurrences pour tenter de définir le LN sont plus grands (12 définitions pour 46 occurrences) chez les apprenants de première année que chez leurs pairs des niveaux supérieurs pour lesquels la notion semble moins abstraite. On peut supposer que les novices entament un processus de réflexion et d'objectivation plus intense en émettant davantage d'hypothèses sur cette figure du natif à laquelle les autres générations sont exposées depuis plus longtemps dans leur apprentissage d'un LE. Malgré le fait que les enquêtes savent se déclarer, à l'unanimité, être natifs du slovène (question 10'), ce processus de catégorisation des langues et des locuteurs, nécessaire pour caractériser les contextes interactionnels en DLC, renvoie sans aucun doute « à un idéal constitué par un locuteur natif, normé, monolingue, et en définitive, abstrait » (Mondada, $1999: 21$ ).

Cette difficulté à définir le LN, notamment par un tiers des répondants qui invoquent le sujet enseignant pour définir le sujet parlant (question 10), se retrouve dans la confusion entre LN et EN que font 18,6\% des apprenants (question 11). La prégnance de cette perception pourrait s'expliquer du fait de la terminologie employée dans le questionnaire slovène. En effet, nous avons dû recourir à l'anglais pour garantir la compréhension du syntagme slovène (naravni oz. rojeni govorec). Or, " native-speaker » est le syntagme fréquemment utilisé par les enseignants slovènes pour faire référence à l'EN, notamment quand ils font la promotion des langues étrangères et de la présence d'un ou plusieurs EN dans leur établissement scolaire. De plus, la figure du « native-speaker » étant en quelque sorte incarnée par l'EN - et lui seul, dans cet établissement secondaire - ce pourrait être ce glissement lexical qui pousse les apprenants, en particulier ceux de deuxième année $(31,6 \%)$ alors en pleine de renégociation de leurs répertoires linguistiques propres à ce niveau d'apprentissage, à confondre locuteur et enseignant. Les quatrièmes années, en revanche, forts de leurs enseignements acquis et de leur parcours d'apprentissage ont complètement objectivé la distinction LN/EN.

Pour justifier la différence entre LN et EN, les répondants invoquent non seulement les compétences linguistiques $(15,3 \%)$ mais surtout ils mettent en valeur la compétence professionnelle de l'EN (54,3\%). Faisons remarquer que l'on observe une plus grande adhésion à cette compétence au fur et à mesure que l'on progresse dans l'apprentissage. Les niveaux supérieurs sont plus enclins à reconnaître la légitimité professionnelle de l'EN (63,2\%) que les niveaux débutants (50\%). Cette valence repose sur le présupposé que l'EN est un meilleur professeur parce qu'il « connaît mieux les secrets de la langue et les stratégies pour y accéder ». Cette forte corrélation entre les compétences linguistique et professionnelle est objectivée par 9,5\% des apprenants, et plus fortement par les apprenants de la 1 ère année $(16,7 \%)$ qui tendent à confondre LN et EN, probablement parce qu'ils idéalisent le locuteur natif prétendument capable d'enseigner sa langue maternelle 
(Davies, 2003 et 2013). Or, pour la majorité des apprenants, l'EN « est préparé pour enseigner à l'école» et «plus compétent» que le LN « qui ne sait pas enseigner ». Ainsi, cette légitimité d'enseignement repose également sur la compétence linguistique de l'EN a priori supérieure à celle du LN. Elle est définie en termes de prononciation, d'intelligibilité de la langue orale, de richesse lexicale, de contrôle des structures grammaticales, de maîtrise des variétés et des registres de langues. Tandis que le LN fonctionne de façon intuitive, sans distanciation par rapport à l'objet linguistique : le $\mathrm{LN}$ « ne réfléchit pas au fonctionnement de la langue quand il parle », « il utilise les particularités de la langue de façon automatique et inconsciente ». Il s'agit donc de deux légitimités différenciées déjà identifiés plus haut : sujet parlant - simple usager de sa langue maternelle qu'il traite de façon intuitive - et sujet enseignant - expert et modèle de langue/culture, objet par rapport auquel il est capable de se distancier pour pouvoir l'enseigner.

Si la distinction entre locuteur et enseignant natif n'est pas toujours évidente, les discours des apprenants ont fait apparaître la prégnance du modèle incarné par le natif et son idéalisation. Notre hypothèse de départ stipulait un changement de représentations en fonction des niveaux d'apprentissage, ce qui se confirme grâce au positionnement tranché des apprenants du niveau supérieur en faveur de l'EN auquel ils attribuent à l'unanimité des compétences enseignantes que le $\mathrm{LN}$ ne peut posséder sans formation adéquate. Les autres générations, et en particulier les débutants - exposés depuis peu à la rencontre pédagogique avec l'EN - n'ont pas encore objectivé la distinction locuteur/enseignant. Pour eux, le natif représente avant tout un informateur, une ressource d'apprentissage (Cuq, $2003:$ 160).

\subsection{Représentations de l'enseignant natif : le constat d'une « valeur ajoutée »}

Si le point de départ de cette enquête portait sur la prétendue « valeur ajoutée » de l'EN, force est de constater que les apprenants adhèrent unanimement à l'affirmation selon laquelle « apprendre une langue étrangère avec un enseignant natif est une valeur ajoutée » (question 12, item f). Cette adhésion totale complète les évaluations soumises aux apprenants sur le tandem EN/ENN. Celles-ci s'assimilaient à des stéréotypes - fonctionnant comme des « prêt-à-parler » pour permettre l'intercompréhension (Moore, 2012 :16) - communément attribués à chaque enseignant et amenaient les enquêtés à juger de la véracité de ces stéréotypes sur la qualité de l'enseignement (items a, b, d, f), des savoirs de l'enseignant sur langue cible (item e) et des connaissances de la culture cible (item c). Ainsi, les représentations se révèlent très fortement ancrées et diffuses entre les niveaux : $94,4 \%$ estiment que «l'on apprend mieux une langue étrangère avec un professeur natif » (item a), « l'enseignant natif enseigne mieux la langue et sa prononciation » $(99,5 \%$, item b) et enfin, " l'enseignant natif connaît mieux la culture et la civilisation de son 
pays d'origine » $(100 \%$, item c). En contrepoint, 53\% des interrogés réfutent l'idée selon laquelle « l'enseignant non-natif enseigne mieux la langue étrangère car il l'a lui-même apprise », et parmi eux, un nombre non négligeable d'apprenants de première $(70,5 \%)$ et de troisième année $(60,7 \%)$ idéalise davantage l'EN que leurs pairs.

On peut donc supposer que ce n'est pas l'expérience particulière de nos apprenants avec un EN, qui leur fait penser qu'ils « apprennent mieux » à son contact. Ce serait le discours diffus, social ou institutionnel, sur « la valeur ajoutée de l'EN » qui justifierait l'existence d'un tel stéréotype l'inscrivant dans l'imaginaire collectif (Py \& Oesch-Serra, 1993 et Oesch-Serra \& Py, 1997). Cela illustre le caractère figé des stéréotypes dont la pertinence pratique dans le discours est due essentiellement à leur potentiel simplificateur et donc univoque, à une stabilité rassurante et intelligible pour les membres du groupe. Pour parvenir à un positionnement, la définition de Mannoni (2010 : 30) constitue un support solide pour saisir la portée de ces constructions mentales dans le discours des apprenants face à l'EN. Ainsi, le stéréotype serait « la rationalisation du préjugé [lequel] semble se tenir beaucoup plus près de l'attitude ». Si l'on suit son raisonnement, afin de déterminer la manière de considérer l'EN dans un contexte d'enseignement/apprentissage alloglotte, le préjugé serait la survalorisation de l'EN, et le stéréotype, les justifications que l'on donne à cette attitude : l'EN s'exprime de façon naturelle et innée dans sa langue maternelle qu'il enseigne, il est donc un meilleur enseignant. Dès lors, la représentation se placerait en amont du stéréotype, dans la mesure où elle constituerait le filtre déformant et simplifiant le réel qui provoque l'attitude et, partant, les justifications stéréotypées.

D'autre part, l'affect et la motivation jouent un rôle prépondérant dans la valorisation du cours avec l'EN : 69\% affirment se sentir « à l'aise » (question 15) avec l'EN dont ils apprécient particulièrement les qualités humaines (question 15'), le contact privilégié (question 17) et l'approche pédagogique (question 21) : le cours est qualifié de «varié, amusant, dynamique, intéressant, riche, différent ». Les apprenants, tous niveaux confondus, se sentent « intégrés à la communication en langue étrangère », " en contact direct avec un locuteur natif ». Cette immersion linguistique est vécue comme un moment d'apprentissage agréable pendant lequel l'EN « fait vivre la langue de façon familière et authentique » en s'éloignant temporairement de son rôle d'enseignant - les rapports qu'ils entretiennent avec lui sont qualifiés de « détendus » $(38 \%)$ et « chaleureux » $(18,6 \%)-$ d'ailleurs, l'EN « enseigne mieux la communication authentique » $(99,2 \%$, question 13 , item f).

Les apprenants débutants apprécient «le contact direct avec un locuteur natif qui permet de communiquer constamment en langue étrangère » et l'usage de langue " pratique et authentique ». À un niveau intermédiaire, le cours avec l'EN est saisi comme « une rencontre, une opportunité, un changement » dans la routine des cours scolaires, et le cours de FLE est vécu comme un bain linguistique actif « où l'on échange en français de façon inconsciente ». Pour le niveau avancé, la communication exclusive en langue 
étrangère « force à fournir plus d'efforts pour parler et pratiquer la langue ». En outre, ces conversations en LE sont l'occasion d'envisager, en la simulant, la rencontre avec l'Autre, le natif pris dans son contexte naturel : "Quand on discute avec l'enseignant natif, on peut imaginer ce que ce serait de parler français si on visitait un pays francophone. » De plus, ils apprécient de « connaître le point de vue de l'EN sur sa propre culture » et de partager leurs « opinions dans des discussions plus ouvertes et spontanées ». Ici, l'enseignant-natif-étranger apporte « un degré de réalité supplémentaire » (Zarate, 1993 : 10), abolissant la distance géographique et imaginaire entre l'apprenant et l'Autre, et constitue un vecteur de motivation pour dynamiser l'apprentissage.

\subsection{Perceptions du tandem EN/ENN : des modèles diversifiés}

La question 13 mettait en regard les compétences linguistiques et enseignantes de l'EN et de l'ENN - linguistique (items a et b : expertise, maîtrise, connaissance de la langue/ culture cible), métalinguistique (items c : explication des savoirs grammaticaux, lexicaux, orthographiques, liés à la phonétique et à la prononciation), sociolinguistique (item e) et la compétence d'évaluation (items f et $\mathrm{g}$ : décentration et correction) - les apprenants devaient attribuer une compétence à l'un ou l'autre enseignant. Le croisement des analyses quantitative et qualitative présente des objectivations assez homogènes, et l'on distingue d'emblée deux modèles diversifiés pour chaque enseignant : l'EN comme modèle de langue/culture et l'ENN comme modèle d'apprentissage.

\subsubsection{L'EN : modèle de langue/culture}

Si l'EN « maîtrise mieux la langue qu'il enseigne » $(96,9 \%)$, il est avant tout un modèle pour la prononciation qu'il « explique mieux » $(97,7 \%)$, tout comme la phonétique (91,5\%). Les apprenants décrivent la prononciation tantôt dans la relation d'usage de la langue ( $\mathrm{L}^{\prime} \mathrm{EN}$ a une bonne prononciation »), tantôt dans la relation d'apprentissage («L'EN peut m'aider à améliorer ma prononciation par la pratique »). La prononciation « authentique » est le gage sécurisant d'une bonne intercompréhension dans la relation apprenants/EN et dans la relation entre pairs. Les niveaux débutants de première et deuxième années sont particulièrement sensibles à la clarté de la prononciation de l'EN qui leur permet de « mieux comprendre ce qui se dit et comment ça se dit » en langue étrangère. Le souci d'une " bonne prononciation » peut néanmoins compromettre la relation d'apprentissage et susciter l'appréhension de s'exprimer en LE : « Je ne me sens ni à l'aise ni mal à l'aise parce que j'ai du mal à prononcer ». Dans ce cas-là, le modèle de prononciation incarné par l'EN renvoie l'image d'un idéal inaccessible qui peut décourager l'apprenant (Davies 2003), surtout à un niveau débutant. Toutefois, la prononciation permettrait d'entrer pleinement en contact avec la langue/culture cible. En imaginant la déperdition d'un tel modèle de prononciation en classe de FLE, les apprenants débutants craignent 
de « perdre le contact avec la vraie prononciation » autrement dit, de s'éloigner de ce qui représente pour eux la norme incarnée par le natif.

Par ailleurs, l'EN « connaît mieux les expressions courantes » $(93,8 \%)$, « les usages de la langue » $(91 \%)$ et « les aspects culturels » $(99,2 \%)$, « il corrige mieux mes fautes de langue étrangère » $(77,2 \%)$; ces représentations diffuses font de lui un modèle authentique de langue/culture. La compétence culturelle dans la relation d'apprentissage est assez peu définie et s'exprime plus précisément à un niveau supérieur d'apprentissage. Les apprenants débutants sont sensibles au degré de proximité de la langue/culture cible que crée la rencontre avec l'EN, vécue comme une « expérience authentique et rapprochée » qui rendrait les contenus d'enseignement «plus fiables ». Les apprenants plus exposés à des contenus culturels - en troisième et quatrième année de la "Classe européenne » par exemple, ou encore ceux qui préparent la matura ${ }^{7}$ de français - déclarent le plus souvent que « l'EN connaît mieux la culture et la civilisation de son pays d'origine » - et l'on devine en amont des attitudes des apprenants, une forte corrélation entre la compétence culturelle « authentique » de l'EN et sa légitimité d'enseignement pour ce type de contenu : comme l'EN connaît sa langue et sa culture, il sait aussi les enseigner, il en est donc le seul modèle de référence disponible, la seule incarnation. Comme le précise Derivry-Plard (Dervin \& Badrinathan, 2011 : 87-88), « ce type de raisonnement (...) démontre en partie la pauvreté de la pédagogie/éducation interculturelle ou à la dimension éducative à l'altérité en situation scolaire ou non scolaire où la culture tout comme la langue sont, en partie, perçues comme des attributs de type essentialiste : l'enseignant « natif » a l'accent, il est l'accent, l'enseignant « natif » a la culture, il est la culture ! ».

\subsubsection{L'ENN : modèle d'apprentissage}

Lorsque les apprenants sont invités à comparer d'une part, les rapports entretenus avec l'EN et l'ENN, et d'autre part, leurs façons d'enseigner, le recours à la langue maternelle est immédiatement repérable. En effet, il représente une sécurité linguistique pour les apprenants moins à l'aise dans la relation d'apprentissage de la LE : «Avec l'ENN, je me sens plus à l'aise parce que je peux m'exprimer en slovène et donner mon avis ». À tous les niveaux d'apprentissage, les apprenants reconnaissent que l'ENN est un gage de sécurité pédagogique car il possède une compétence d'enseignement légitimée par quatre avantages visà-vis de l'EN : il connaît mieux les difficultés de la langue cible $(84,3 \%)$, il est conscient des difficultés d'apprentissage en LE (83\%), il explique mieux la grammaire $(79,6 \%)$ et l'orthographe $(58,6 \%)$. L'ENN peut avoir recours à la comparaison interlinguale pour développer une réflexion métalinguistique et accompagner l'apprenant dans la construction de son répertoire linguistique : «L'ENN peut m'aider à résoudre mes problèmes d'apprentissage, corriger mes fautes, comprendre le fonctionnement de la langue étrangère, traduire ou expliquer en langue maternelle, comparer les systèmes linguistiques ». L'ENN est un

7 Cf. note 4. 
médiateur qui permet de faire le lien entre langue/culture source et langue/culture cible par comparaison et avec lequel on apprend à se décentrer : « Il est possible de commenter la culture étrangère en partant d'un même point de vue pour se forger une opinion ». Le modèle d'apprentissage incarné par l'ENN et son rôle de médiateur/accompagnateur dans le processus d'appropriation de la langue peuvent être considérés comme « une forme particulière d'interaction de tutelle au sens où l'entend Bruner» (Causa 2012: 58). Autrement dit, le recours à la LM, permet à l'ENN de prendre en charge, d'étayer les éléments de la tâche que l'apprenant ne peut pas réaliser seul en LE.

L'ENN incarne un modèle d'apprentissage sécurisant à tous les niveaux : « Avec l'ENN, il est possible d'expliquer des aspects de la langue étrangère qui sont évidents pour l'enseignant natif mais pas pour nous, de parler slovène pour (se faire) comprendre, de comprendre les explications sur la langue étrangère en langue maternelle ». L'analyse métalinguistique en LM est un processus d'apprentissage que les apprenants estiment nécessaire pour dépasser leur sentiment d'insécurité linguistique. La prise de distance par rapport au processus d'appropriation fait remarquer à ces trois élèves de quatrième année qu'avec l'ENN, « il est plus facile de débuter l'apprentissage d'une langue », ce qu'aucun débutant n'a conscientisé, probablement par manque de distanciation. Le caractère sécurisant de l'ENN apparaît de façon implicite dans les dires des apprenants sur les rapports entretenus avec l'EN. Un apprenant de troisième année déclare : « Avec l'EN, je me sens mal à l'aise parce que je ne peux pas communiquer avec lui en slovène et souvent je ne comprends pas ses explications ». Lorsque les contenus d'enseignement se complexifient, les apprenants ont besoin d'un point d'ancrage dans le connu (la langue/ culture source) pour se rassurer. D'ailleurs, d'aucuns considèrent qu'il est plus facile de s'identifier à l'ENN car il est passé par l'apprentissage de la langue. Il est conscient des obstacles d'apprentissage de la LE et peut les guider dans le processus d'apprentissage en ayant recours à la LM qui représente une sécurité linguistique. Toutefois, ils estiment que l'approche plus méthodique et plus systématique de l'ENN pour expliciter des contenus grammaticaux en ayant éventuellement recours à la LM, les rendrait plus passifs, alors que l'approche « ludique et intéressante » de l'EN qui interagit exclusivement en LE avec eux, leur donne l'impression d'être plus actifs dans le processus d'apprentissage. En effet, la moitié des apprenants (53\%) estime que le fait qu'il soit passé lui-même par l'apprentissage de la langue cible ne garantit pas qu'il l'enseigne mieux. L'ENN constitue donc un modèle d'apprentissage mais pas forcément un modèle d'enseignement.

Dans ce contexte d'enseignement, il apparait clairement que la confrontation de ces deux modèles diversifiés s'est construite en fonction des positions différentielles entre EN et ENN (Derivry-Plard 2011). Comme le résume cet apprenant de quatrième année de façon schématique : "l'ENN enseigne la théorie qu'on met en pratique avec l'EN ». Ces représentations instrumentalisent les enseignants et s'inscrivent pleinement dans des schèmes de perception réifiants qui perpétuent des modèles d'apprentissage difficiles à désincarner. 
L'étude comparative par niveaux d'apprentissage a révélé l'objectivation de catégories de perceptions qui valident d'une part, la force du modèle incarné par le natif, d'autre part, la prégnance de deux légitimités sociales d'enseignement réparties entre EN (modèle de langue) et ENN (modèle d'apprentissage). L'enquête a également permis de dégager les critères à partir desquels apprendre une langue étrangère avec un enseignant natif constituerait un atout ; il s'agit en fait des trois critères que Coulmas (1981) attribue au natif: « authenticité, fiabilité, représentativité », ce qui confirme que la légitimité du natif, locuteur ou enseignant, n'est pas remise en question. Par ailleurs, il est possible de repérer des attitudes et des comportements d'apprentissage sensiblement marqués aux deux extrêmes de l'échelle des niveaux d'apprentissage : les débutants se focalisent davantage sur l'optimisation de leurs compétences à l'oral, et les confirmés, sur le développement de leurs capacités de réflexion par rapport à la langue/culture cibles. Quant aux niveaux intermédiaires, les résultats de l'analyse sont plus complexes. Pour gérer le degré de sécurité/insécurité linguistique ressentie dans la relation d'apprentissage ou d'usage de la langue, ils sélectionnent les modèles d'enseignant qui correspondent à leurs besoins; ils privilégient l'un ou l'autre enseignant en fonction de leurs habitudes d'apprentissage et des contenus d'enseignement qui les exposent plus ou moins fréquemment à l'EN. Tout compte fait, le natif est idéalisé par l'ensemble des apprenants parce qu'il leur est difficile à définir, d'une part, et il est intelligible, rassurant pour eux de le réduire à un modèle de compétence monolingue à imiter, d'autre part.

Évidemment, l'enseignement dans ce contexte institutionnel précis correspond en fait à une répartition " compartimentalisée " des contenus selon le profil des enseignantes (Dabène 1990 ; Causa 2002 ; Derivry-Plard 2008 ; Dervin 2011 ; Dervin \& Badrinathan (dir.) 2011) ; en effet, les contenus linguistiques (grammaire, lexique, orthographe, phonétique) sont ici assignés à l'ENN qui suit la progression d'un programme imposé à l'aide d'un manuel d'apprentissage, et les contenus culturels et sociolinguistiques (tâches communicatives d'expression orale, le plus souvent, que les apprenants perçoivent également comme des activités de prononciation) reviennent à l'EN qui intervient à intervalles réguliers, en fonction des contenus abordés en classe par l'ENN. Les apprenants étant habitués à ce que les contenus soient répartis ainsi, le tandem joue finalement en défaveur de l'ENN et le constat d'une « valeur ajoutée » attribuée à l'EN fait apparaître la survalorisation de son statut sociolinguistique profondément ancrée dans la culture éducative des apprenants. Le recueil des données affectives témoigne de l'enthousiasme que suscite la présence de l'EN pour des contenus plus " intéressants et amusants », et les perceptions du cours de FLE sans EN décrivent une déperdition de qualité des contenus et des savoirs transmis. Ce qui amène la question suivante : ces modèles de langue/ culture et d'apprentissage cesseraient-ils d'être confortés si les rôles des enseignantes et les contenus qu'elles enseignent étaient inversés ? Un tel dispositif suffirait-il à faire voler 
en éclats l'opposition EN/ENN et défaire les apprenants du poids des représentations apparues dans cette analyse comparative ?

C'est pourquoi, nous envisageons, dans le prolongement de cette étude, interroger des étudiants de FLE dans l'enseignement supérieur, pour déterminer la force des représentations à l'égard du locuteur natif et du couple EN/ENN auquel ils sont exposés tout au long de leur cursus universitaire. L'échantillon serait constitué des étudiants de licence et de master de filières différentes, pédagogique et linguistique. Étant donné leur niveau de compétence linguistique avancé, leur profil plurilingue - forgé notamment par l'étude d'une autre langue étrangère pour certains - et l'exposition constante à une variété de contenus d'enseignements dispensés par des enseignants aux statuts sociolinguistiques composites, nous supposons que cette communauté d'apprenants conscientisent plus fortement son apprentissage et légitiment davantage les compétences de transmission (la technè), d'enseignement et d'éducation en langues-cultures (Cook, 1999, 2001 ; Ellis 2006 in Derivry-Plard 2011) de leur professeurs. Il faudrait probablement améliorer le recueil des données - soit en soumettant des questionnaires plus exhaustifs, soit sous forme d'entretiens semi-directifs, en ciblant notamment sur les contenus d'enseignement - et procéder à une analyse plus rigoureuse d'après les méthodologies de Bardin (1993) et Martin (2007) sans négliger l'apport de la sociologie (Derivry-Plard 2014) dans le champ de la didactique des langues-cultures étrangères qui est le nôtre.

\section{BIBLIOGRAPHIE}

ABRIC, Jean-Claude (1994) Les représentations sociales. Paris : PUF.

BARDIN, Laurence (1993) L'analyse de contenu. Paris : PUF.

BEACCO, Jean-Claude/Jean-Louis CHISS/Francine CICUREL/Daniel VÉRONIQUE (dir.) (2005) Les cultures éducatives et linguistiques dans l'enseignement des langues. Paris : PUF.

BOGAARDS, Paul (1991) Aptitude et affectivité dans l'apprentissage des langues étrangères. Paris : Hatier, coll. LAL.

CASTELLOTTI, Véronique/Danièle MOORE (2002) Représentations sociales des langues et enseignements. Strasbourg : Conseil de l'Europe, Division des politiques linguistiques. http://www.coe.int/t/dg4/Linguistic/Source/CastellottiMooreFR.pdf.

CAUSA, Marina/Monica VLAD (2008) Être enseignant de langues à l'heure européenne : analyse comparée des représentations, croyances et savoirs des futurs enseignants de langues. Perspectives pour une didactique des langues contextualisée. CIRDL AUF, Éditions des Archives Contemporaines, 115-129.

CAUSA, Marina (2009) Discipline non linguistique. Le statut sociolinguistique du professeur. Le Français dans le monde, $\mathrm{n}^{\circ}$ 361, janvier-février 2009, 26-27.

COULMAS, Florian (1981) A Festschrift for native speaker. La Haye : Mouton. 
CUQ, Jean-Pierre (dir.) (2003) Dictionnaire de didactique du français. Langue étrangère et seconde. Paris : CLE International, coll. Asdifle.

DABÈNE, Louise (dir.) (1990) Variations et rituels dans la classe de langue étrangère. Paris : Hatier, coll. LAL.

DABÈNE, Louise (1994) Repères sociolinguistiques pour l'enseignement des langues. Paris : Hachette, coll. F.

DAVIES, Alan (2003) The Native speaker: myth and reality. Clevedon : Multilingual matters.

DAVIES, Alan (2013) Is the native speaker dead? Histoire Épistémologie Langage, tome 35, fascicule 2, 17-28. www.persee.fr/doc/hel_0750-8069_2013_num_35_2_3455.

DE PIETRO, Jean-François/Nathalie MULLER (1997) La construction de l'image de l'autre dans l'interaction. Des coulisses de l'implicite à la mise en scène. Bulletin VALS/ASLA 65, Communication et pragmatique interculturelles, 25-46. http://doc. rero.ch/record/20650/files/25-46_De_Pietro_et_Muller.pdf.

DERIVRY-PLARD, Martine (2006) Les enseignants natifs et non-natifs de langue(s) : catégorisation linguistique ou construction sociale ? Travaux de didactique du FLE, $\mathrm{n}^{\circ}$ 55, 100-108. http://www.academia.edu/3487687/Les_enseignants_natifs_et_ non-natifs_de_langue_s_categorisation_linguistique_ou_construction_sociale.

DERIVRY-PLARD, Martine (2008) Enseignants « natifs » et « non-natifs » : deux profils professionnels en concurrence sur le marché des langues. G. Zarate, D. Lévy et C. Kramsch, Précis du plurilinguisme et du pluriculturalisme. Paris : Éditions des Archives contemporaines, 189-191.

DERIVRY-PLARD, Martine (2014) Recherche en didactique des langues : la perspective sociologique et le quantitatif oubliés ? Recherche et pratiques pédagogiques en langues de spécialité, Vol. XXXIII, n² 2. http://journals.openedition.org/apliut/4396.

DERVIN, Fred/Vasumathi BADRINATHAN (dir.) (2011) L'enseignant non natif : identités et légitimité dans l'enseignement-apprentissage des langues étrangères. Bruxelles : E.M.E., coll. Proximités didactiques.

DE SINGLY, François (2005) Le questionnaire. (Série « L'enquête et ses méthodes »). Paris : Armand Colin.

GRIFFIN, Claire (2012) L'identité professionnelle des professeurs d'anglais "locuteurs natifs » exerçant en France depuis le traité de Maastricht. Entre conservation ontologique et acculturation : les limites du capital natif. Thèse de doctorat en didactologie des langues et des cultures. Paris : Université Sorbonne nouvelle - Paris 3.

HOLLIDAY, Adrian (2006) Native-speakerism. ELT Journal 60/4, 385-387. http://eltj. oxfordjournals.org/content/60/4/385.full.

JODELET, Denise (dir.) (1989) Les représentations sociales. Paris : PUF.

LAMY-JOSWIAK, Anne-Cécile (2013) Représentations de l'enseignant natif chez les apprenants slovènes. Mémoire de Master en didactique du français et des langues. Paris : Université Sorbonne nouvelle - Paris 3. 
MANNONI, Pierre (2010) Les représentations sociales. Paris : PUF, coll. « Que sais-je ? ».

MARTIN, Olivier (2007) L'analyse de données quantitatives. Paris : Armand Colin.

MEDGYES, Peter (1992) Native or non-native: who's worth more ? ELT Journal 46/4, 340-349. http://203.72.145.166/ELT/files/46-4-2.pdf.

MONDADA, Lorenza (1999) L'accomplissement de « l'étrangéité » dans et par l'interaction : procédures de catégorisation des locuteurs. Langages, $33^{\text {ème }}$ année, $\mathrm{n}^{\circ}$ 134, 20-34. http://www.persee.fr/web/revues/home/prescript/article/ lgge_0458-726X_1999_num_33_134_2190.

MOORE, Danièle (dir.) (2012) Les représentations des langues et leur apprentissage : références, modèles, données et méthodes. Paris : Didier, coll. « Crédif-Essais ».

MOSCOVICI, Serge $(1961,1976)$ La psychanalyse : son image et son public. $2^{\mathrm{e}}$ éd. rév. Paris : PUF.

OESCH-SERRA, Cecilia/Bernard PY (1997) Le crépuscule des lieux communs, ou les stéréotypes entre consensus, certitude et doute. TRANEL, 27, 29-49.

PAIKEDAY, Thomas M. (1985) The Native Speaker is Dead! An informal discussion of a linguistic myth with Noam Chomsky and other linguists, philosophers, psychologists, and lexicographers. Toronto; New York : Paikeday Publishing Inc.

PY, Bernard (2004) Pour une approche linguistique des représentations sociales. J.-C. Beacco (dir.), Représentations métalinguistiques ordinaires et discours, Langages, $\mathrm{n}^{\circ} 154,6-19$.

PY, Bernard/Cecilia OESCH-SERRA (1993) Dynamique des représentations dans des situations de migration. Étude de quelques stéréotypes. Bulletin CILA, 57, 71-83.

RENAUD, Patrick (1998) Absoute pour un locuteur natif. Le français en Afrique, $\mathrm{n}^{\circ} 12$. http://www.unice.fr/ILF-CNRS/ofcaf/12/12.html.

ZARATE, Geneviève (1993) Représentations de l'étranger et didactique des langues. Paris : Didier, coll. « Crédif-Essais ».

ZARATE, Geneviève/Maurice CANDELIER (2002) Les représentations en didactique des langues et cultures. Notions en questions 2/1997. Paris : Didier Érudition. 


\section{ANNEXE : QUESTIONNAIRE}

\section{Bonjour!}

Dans le cadre d'une recherche en didactique du français et des langues, j'effectue une recherche sur la supposée "valeur ajoutée » de l'enseignant natif en cours de français langue étrangère. Vos réponses me permettront de vérifier la véracité de l'hypothèse selon laquelle on apprendrait mieux une langue étrangère avec un enseignant natif.

Merci de votre participation.

\section{Toi et l'apprentissage du FLE}

1. Âge :

2. Niveau d'études (année scolaire en cours) :

$1^{\text {ère }}$ année o $\quad 2^{\text {ème }}$ année o $\quad 3^{\text {ème }}$ année o $\quad 4^{\text {ème }}$ année o $\quad$ Matura de français o

3. Français : LV2 o LV3 o

4. Matières :

FLE o Culture et civilisation (CC) o Cours de préparation à la Matura $\mathrm{o}$

5. Si tu as passé l'examen du DELF Junior, indique ton niveau :
A1 o
A2 o
B1 o

6. Nombre d'heures de cours de français par semaine :

o 3 heures

o 4 heures (3h FLE $+1 \mathrm{~h} \mathrm{CC} 4^{\text {ème }}$ année)

o 5 heures ( $3 \mathrm{~h} \mathrm{FLE}+2 \mathrm{~h} \mathrm{CC} 3^{\text {ème }}$ année) ou ( $3 \mathrm{~h} \mathrm{FLE}+2 \mathrm{~h}$ Matura de français niveau de base/supérieur)

o 6 heures ( $3 \mathrm{~h} \mathrm{FLE}+1 \mathrm{~h} \mathrm{CC} 4^{\text {ème }}$ année $+2 \mathrm{~h}$ Matura de français niveau de base)

o 8 heures ( $3 \mathrm{~h} \mathrm{FLE}+1 \mathrm{~h} \mathrm{CC} 4^{\text {ème }}$ année $+2 \mathrm{~h}$ Matura de français niveaux de base et supérieur)

7. Nombre d'heures de cours de français avec l'enseignant natif :

o 1 heure par semaine

o 1 heure tous les quinze jours

o 2 heures par semaine

o 3 heures par semaine

o 3 heures tous les quinze jours

o 4 heures par semaine

8. Avant d'entrer au lycée, avais-tu déjà suivi des cours de langue étrangère avec un enseignant natif ?

OUI o NON o Si oui, pour quelle(s) langue(s)? 
9. Actuellement, suis-tu des cours de langue étrangère avec un autre enseignant natif?

OUI o NON o

\section{Le locuteur natif}

10. Pour toi, qu'est-ce qu'un locuteur natif ?

10'. Pour quelle(s) langue(s) te considères-tu être un locuteur natif ?

11. Penses-tu qu'il existe une différence entre un locuteur natif et un enseignant natif ? OUI o NONo Si oui, laquelle?

Les enseignants de langue étrangère, natif et non natif

12. Lis les définitions suivantes et indique $(X)$ si elles sont vraies ou fausses.

\begin{tabular}{|l|l|l|}
\hline Affirmations & Vrai & Faux \\
\hline a) On apprend mieux une langue étrangère avec un professeur natif. & & \\
\hline b) L'enseignant natif enseigne mieux la langue et sa prononciation. & & \\
\hline $\begin{array}{l}\text { c) L'enseignant natif connaît mieux la culture et la civilisation de son pays } \\
\text { d'origine. }\end{array}$ & & \\
\hline $\begin{array}{l}\text { d) L'enseignant non natif enseigne mieux la langue étrangère car il l'a } \\
\text { lui-même apprise. }\end{array}$ & & \\
\hline e) L'enseignant non natif connaît mieux les difficultés de la langue étrangère. & & \\
\hline $\begin{array}{l}\text { f) Apprendre une langue étrangère avec un enseignant natif est une « valeur } \\
\text { ajoutée ». }\end{array}$ & \\
\hline
\end{tabular}


13. Lis les affirmations ci-dessous, coche $(X)$ ce qui, selon toi, correspond le mieux à l'un des deux enseignants.

\begin{tabular}{|l|l|l|}
\hline Affirmations & Enseignant natif & Enseignant non natif \\
\hline $\begin{array}{l}\text { a) Il est expert de la langue } \\
\text { qu'il enseigne. }\end{array}$ & & \\
\hline $\begin{array}{l}\text { b) II maîtrise mieux la langue } \\
\text { qu'il enseigne. }\end{array}$ & & \\
\hline c) Il explique mieux: & & \\
\hline - la grammaire, & & \\
\hline - le vocabulaire, & & \\
\hline - l'orthographe, & & \\
\hline - la prononciation, & & \\
\hline - la phonétique. & & \\
\hline d) Il connaît mieux: & & \\
\hline - les expressions courantes, & & \\
\hline - les usages de la langue, & & \\
\hline - les aspects culturels. & & \\
\hline $\begin{array}{l}\text { e) Il enseigne mieux } \\
\text { la communication } \\
\text { authentique. }\end{array}$ & & \\
\hline $\begin{array}{l}\text { f) Il est conscient de mes } \\
\text { en langue étrangère. }\end{array}$ & & \\
\hline $\begin{array}{l}\text { g) Il corrige mieux mes fautes } \\
\text { de langue étrangère. }\end{array}$ & & \\
\hline
\end{tabular}

14. Complète les phrases suivantes : $(\mathrm{EN}=$ enseignant natif; $\mathrm{ENN}=$ enseignant non natif)

a) L'EN peut m'aider à

b) L'ENN peut m'aider à

c) Avec l'EN, il est plus facile de

et il est possible de

d) Avec l'ENN, il est plus facile de

et il est possible de

e Suivre des cours avec l'EN me permet de 


\section{Tes rapports avec l'enseignant natif}

15. Comment te sens-tu pendant les cours dispensés par l'EN ? Coche (3) l'expression qui te correspond le mieux.

\begin{tabular}{|l|l|l|l|l|}
\hline Très à l'aise & À l'aise & Ni à l'aise, ni mal à l'aise & Mal à l'aise & Très mal à l'aise \\
\hline & & & & \\
\hline
\end{tabular}

15'. Pourquoi te sens-tu comme ça?

16. Est-ce que tes rapports avec l'EN sont différents de ceux que tu entretiens avec l'ENN ?

\begin{tabular}{|l|l|l|}
\hline OUI & NON & SANS OPINION \\
\hline & & \\
\hline
\end{tabular}

16'. Peux-tu expliquer pourquoi?

17. Comment peux-tu qualifier tes relations avec l'enseignant natif?

Coche (3) le ou les adjectifs qui te semblent approprié(s).

\begin{tabular}{|l|l|l|l|l|l|l|l|}
\hline Distantes & Proches & Amicales & Chaleureuses & Tendues & Détendues & Riches & Inexistantes \\
\hline & & & & & & & \\
\hline
\end{tabular}

\section{Les cours avec l'enseignant natif}

18. Dans les cours de LE dispensés par l'enseignant natif, qu'apprécies-tu particulièrement?

19. D'après ton expérience d'apprentissage avec les deux enseignants - natif et non natify a-t-il, selon toi, des différences dans leur façon d'enseigner une langue étrangère ?

OUI o NON o Si oui, lesquelles? 
20. Comment décrirais-tu ton apprentissage d'une langue étrangère sans enseignant natif?

\section{POVZETEK}

\section{Predstave slovenskih dijakov o učitelju - domačem govorcu}

Prispevek se osredotoča na pomen podobe učitelja tujega jezika - domačega oz. priučenega govorca pri slovenskih dijakih, ki obiskujejo pouk francoščine kot tujega jezika (FLE). Da bi obogatili učenje tujih jezikov, so v Sloveniji uvedli sistem timskega poučevanja, pri katerem naj bi učitelja - domači in priučeni govorec tujega jezika - v razredu izmenjaje poučevala vsebine, povezane $\mathrm{z}$ jezikom in kulturo. $\mathrm{V}$ tem timskem pedagoškem projektu naj bi domači govorec predstavljal dodano vrednost. Pri tem se postavlja vprašanje, kako učenci, ki jih poučuje ta edinstvena dvojica učiteljev, gledajo na učni proces in na svoja učitelja tujega jezika glede na njun sociolingvistični položaj. Cilj naše raziskave je bil prepoznati kriterije, po katerih dijaki oblikujejo svoje mnenje o učitelju tujega jezika. V ta namen smo sestavili vprašalnik, v katerem so dijaki opisali podobo, ki so si jo izoblikovali o učitelju - domačem govorcu francoščine, v primerjavi s podobo, ki so si jo ustvarili o učitelju - priučenem govorcu francoščine. Predvidevali smo, da bo podoba učitelja odvisna od jezikovne ravni dijakov. Izvedli smo primerjalno analizo, saj učenci z učiteljem - domačim govorcem jezika zaradi drugačnih oblik in vsebin poučevanja oblikujejo poseben odnos. Kvantitativna in kvalitativna analiza rezultatov je pokazala, da učiteljev sociolingvistični položaj vpliva na predstave, jezik in učenje dijakov.

Ključne besede : francoščina kot tuji jezik, sociolingvistika, podoba, domači govorec, učitelji tujega jezika

\section{ABSTRACT}

\section{Representations of native-speaker teachers among Slovenian secondary school learners}

This article focuses on the representations of the native/non-native teacher among allophone learners in the context of teaching French as a foreign language (FLE) in Slovenia, where, in order to enrich learning of foreign languages, native-teachers were hired to provide collaborative teaching: native and non-native teachers would rotate language- and culture-based contents to be taught 
consecutively in the classroom. In this context it was believed that the native teacher would represent added value to this team-teaching project. Therefore, the question arises as to how learners, faced with this situation, perceive their learning and foreign language teachers according to their sociolinguistic status. The aim of our research is to identify the criteria for such a perception by asking students about their representations of the native-teacher in comparison to those of their non-native teacher, using a questionnaire. According to our assumptions, these representations would change according to students' language levels. The choice of a comparative study between students' levels was motivated by the special relationship they have with the native teacher, considering their learning habits and teaching contents. Quantitative and qualitative analyses of the results reveal the impact of teachers' sociolinguistic status on the representations of the learners, language and learning.

Keywords: French as a foreign language, sociolinguistics, representations, native-speaker, foreign language teachers 